\title{
Mott-Hubbard metal-insulator transition at noninteger filling
}

\author{
Krzysztof Byczuk, ${ }^{1,2}$ Walter Hofstetter, ${ }^{3}$ and Dieter Vollhardt ${ }^{2}$ \\ ${ }^{1}$ Institute of Theoretical Physics, Warsaw University, Ulica Hoża 69, PL-00-681 Warszawa, Poland \\ ${ }^{2}$ Theoretical Physics III, Center for Electronic Correlations and Magnetism, Institute for Physics, University of Augsburg, \\ D-86135 Augsburg, Germany \\ ${ }^{3}$ Lyman Physical Laboratory, Harvard University, Cambridge, Massachusetts 02138, USA
}

(Received 2 September 2003; published 29 January 2004)

\begin{abstract}
Correlated electrons in a binary alloy $A_{x} B_{1-x}$ are investigated within the Hubbard model and dynamical mean-field theory (DMFT). The random energies $\epsilon_{i}$ have a bimodal probability distribution and an energy separation $\Delta$. We solve the DMFT equations by the numerical renormalization-group method at zero temperature and calculate the spectral density as a function of disorder strength $\Delta$ and interaction $U$ at different fillings. For filling factors $\nu=x$ or $1+x$ the lower or upper alloy subband is half filled and the system becomes a Mott insulator at strong interactions, with a correlation gap at the Fermi level. At the metal-insulator transition hysteresis is observed. We also analyze the effective theory in the $\Delta \rightarrow \infty$ limit and find good agreement between analytical and numerical results for the critical interaction $U_{c}$ at which the metal-insulator transition occurs.
\end{abstract}

DOI: 10.1103/PhysRevB.69.045112

PACS number(s): 71.10.Fd, 71.27. $+\mathrm{a}, 71.30 .+\mathrm{h}$

\section{INTRODUCTION}

A Mott-Hubbard metal-insulator transition (MIT) occurs when the local interaction $U$ between the electrons on a lattice reaches a critical value $U_{c}{ }^{1,2}$ Since in the Mott insulator the particles are essentially localized on lattice sites, the transition can only occur if the number of particles is commensurate with the number of sites, i.e., for integer filling factor $\nu \equiv N / N_{L}$, where $N$ and $N_{L}$ are the number of electrons and lattice sites, respectively. If the translational symmetry of the lattice is broken either spontaneously (as in an antiferromagnet) or by application of some field (as in a superlattice) the unit cell is enlarged and the Mott insulator can, in principle, occur even at a rational filling factor $\nu=p / q$, where $p, q$ $\in \mathbb{N}^{2-4}$ In fact, in our recent investigation of disordered electronic systems ${ }^{5}$ we discovered that in binary-alloy systems $A_{x} B_{1-x}$, composed of two different atoms $A$ and $B$ with concentrations $x$ and $1-x$, respectively, a MottHubbard MIT can even occur for arbitrary noninteger filling factors. This transition takes place if $\nu=x$ or $1+x$, where $0<x<1$. This observation extends the traditional view of the Mott-Hubbard MIT and the notion of Mott insulators to a wider class of systems with, basically, arbitrary fillings. As we will argue below, in such a system magnetic long-range order should be suppressed due to the absence of particlehole symmetry and, therefore, the ground state is likely to be paramagnetic. Therefore, the experimental realization of such a Mott insulator would provide an excellent playground for the study of Mott insulators without long-range order.

In the present paper we study correlated electrons on a lattice using the dynamical mean-field theory ${ }^{6,7}$ (DMFT) applied to the disordered Hubbard model at zero temperature. ${ }^{8}$ The numerical renormalization-group (NRG) method is used to solve the self-consistent DMFT equations. ${ }^{9-12}$ We present results for the single-electron spectral density and the selfenergy to show how a correlated metal away from half filling may turn into a Mott insulator by increasing the disorder. We find hysteresis in this transition, such as the one observed for the MIT in the pure Hubbard model at half filling. ${ }^{13,14}$ From the numerical data we construct a phase diagram at $\nu=x$ and propose a classification scheme for correlated insulators with binary-alloy disorder. We also develop an effective analytic theory which is asymptotically exact in the limit of alloy band splitting and which shows that the Mott-Hubbard MIT occurs at the critical interaction $U_{c} / W \approx 3 \sqrt{x} / 2$, where $W$ is the bandwidth of the noninteracting system.

In Ref. 5 we analyzed the problem of the Mott MIT in a binary-alloy host at relatively high temperatures using the quantum Monte Carlo method to solve the DMFT equations. This only allowed us to detect a crossover from a correlated metal to a Mott insulator. Moreover, since in Ref. 5 we were primarily interested in ferromagnetism in binary-alloy systems, we used the density of states (DOS) corresponding to the fcc lattice in infinite dimension. ${ }^{15}$ Such an unbounded DOS supports ferromagnetism in a one-band Hubbard model, ${ }^{16,17}$ but does not lead to a real gap for the Mott insulator. The NRG method applied here is accurate enough to detect a sharp MIT in a disordered and correlated electron system at zero temperature. Explicit calculations were performed with a semielliptic Bethe DOS, having finite support, which leads to the opening of a genuine gap at the MIT. Additionally, the NRG allows us to determine the self-energy on the real frequency axis and to discuss in detail how the Mott gap opens.

The paper is organized as follows. In Sec. II we introduce the model and discuss the properties of interacting electrons in a binary-alloy host. In Sec. III the DMFT method is introduced to solve the Hubbard model with local disorder. In Sec. IV, we present the numerical results and provide evidence for a MIT away from half filling. In Sec. V an asymptotic theory is developed and in Sec. VI our conclusions are presented. In the Appendix, we present a method to extract the local self-energy within the DMFT in disordered systems. 


\section{ANDERSON-HUBBARD HAMILTONIAN WITH BINARY ALLOY DISORDER}

As a minimal model describing correlated lattice electrons in the presence of disorder we consider the single-orbital Anderson-Hubbard (AH) Hamiltonian

$$
H_{A H}=-t \sum_{\langle i j\rangle \sigma} a_{i \sigma}^{\dagger} a_{j \sigma}+\sum_{i \sigma} \epsilon_{i} n_{i \sigma}+U \sum_{i} n_{i \uparrow} n_{i \downarrow}
$$

where $t>0$ is the hopping integral for the electrons between nearest-neighbor sites, $U$ is the on-site interaction energy between electrons with opposite spins $\sigma= \pm 1 / 2, n_{i \sigma}=a_{i \sigma}^{\dagger} a_{i \sigma}$ is the local electron number operator, and $\epsilon_{i}$ is the local ionic energy which is a random variable. In the following we assume a bimodal probability distribution for the random variable $\epsilon_{i}$, i.e.,

$$
\mathcal{P}\left(\epsilon_{i}\right)=x \delta\left(\epsilon_{i}+\frac{\Delta}{2}\right)+(1-x) \delta\left(\epsilon_{i}-\frac{\Delta}{2}\right),
$$

which corresponds to a binary-alloy system composed of two different atoms $A$ and $B$. The atoms are distributed randomly on the lattice and have ionic energies $\epsilon_{A, B}$, with $\epsilon_{B}-\epsilon_{A}$ $=\Delta$. The parameter $\Delta$ is a measure of the disorder strength. The concentration of $A(B)$ atoms is given by $x=N_{A} / N_{L}$ $\left(1-x=N_{B} / N_{L}\right)$, where $N_{A}\left(N_{B}\right)$ is the number of the corresponding atoms.

From the localization theorem (the HadamardGerschgorin theorem in matrix algebra) it is known that if the Hamiltonian $H_{A H}$, with a bimodal distribution for $\epsilon_{i}$, is bounded, then there is a gap in the single-particle spectrum for sufficiently large $\Delta \gg \max (|t|, U) .{ }^{18,19}$ Hence at $\Delta=\Delta_{c}$ the DOS splits into two parts corresponding to the lower and the upper alloy subbands with centers of mass at the ionic energies $\epsilon_{A}$ and $\epsilon_{B}$, respectively. The width of the alloy gap is of the order of $\Delta$. The lower and upper alloy subbands contain $2 x N_{L}$ and $2(1-x) N_{L}$ states, respectively. If the Hamiltonian is not bounded such as, for example, in the case of a tightbinding Hamiltonian on a hypercubic lattice in infinite dimensions, the alloy gap is reduced to a pseudogap, i.e., the spectral function vanishes only at a single point. These statements hold for all space dimensions. However, the alloy gap can be destroyed by clusters of one type of atoms which are surrounded by atoms of the other type. They create an exponentially vanishing DOS in the gap (Lifshitz tails) near the edges of the alloy subbands. ${ }^{19}$

Binary-alloy disorder in a noninteracting electron system can create two kinds of localized states: (i) states which are localized due to coherent backscattering processes (Anderson localization, Refs. 20 and 21) and (ii) states in the middle of the alloy subbands, ${ }^{22}$ which are localized due to a particular superposition of the electronic wave functions caused by particular arrangements of the alloy atoms. ${ }^{18,23,24}$ While the localized states of type (i) are generic and gradually appear in the alloy subbands starting from the band edges, the localized states of type (ii) can be removed either by a small perturbation of the ionic energies or by an interaction between the particle, and, therefore, are beyond the scope of the present paper.
The most spectacular effect of strong correlations between the electrons in a pure $(\Delta=0)$ system is the Mott-Hubbard metal-insulator transition. It occurs for $\nu=1,2, \ldots, 2 g-1$, where $g$ is an orbital degeneracy, at an interaction $U=U_{c}$ (note that $\nu=2 g$ corresponds to a band insulator). ${ }^{13,25-28}$ For interactions $U \ll U_{c}$ the electrons gain kinetic energy due to the delocalized nature of the wave function. When $U$ increases, the electrons keep at a distance as much as possible, and at $U \geqslant U_{c}$ the many-body wave function is essentially localized at each lattice site. The system is then a Mott insulator. Within the DMFT for Hubbard-like models with single or degenerate orbitals a Mott-Hubbard MIT was shown to occur at integer filling factors $\nu .^{27,28}$ At low but finite temperatures this MIT is discontinuous, while at zero temperature it is continuous, i.e., when approaching the critical interaction from the metallic side the quasiparticle peak continuously narrows until it completely disappears at the transition point. ${ }^{7,13,14}$ In the insulating phase the DOS is zero at the Fermi level and the whole spectral weight is shifted into the two Hubbard subbands, which are remnants of the atomic levels with single and double (multiple) occupancy. Away from integer filling the system is always metallic and the spectral function has visible Hubbard subbands at any finite and large $U$.

New possibilities appear in systems with correlated electrons and binary-alloy disorder. The Mott-Hubbard metalinsulator transition can occur at any filling $\nu=x$ or $1+x$, corresponding to a half-filled lower or to a half-filled upper alloy subband, respectively, as shown schematically for $\nu$ $=x$ in Fig. 1. The Mott insulator can then be approached either by increasing $U$ when $\Delta \geqslant \Delta_{c}$ (alloy band splitting limit), or by increasing $\Delta$ when $U \geqslant U_{c}$ (Hubbard band splitting limit). The nature of the Mott insulator in the binaryalloy system can be understood physically as follows. Due to the high-energy cost of the order of $U$ the randomly distributed ions with lower (higher) local energies $\epsilon_{i}$ are singly occupied at $\nu=x(\nu=1+x)$, i.e., the double occupancy is suppressed. In the Mott insulator with $\nu=x$ the ions with higher local energies are empty and do not contribute to the low-energy processes in the system. Likewise, in the Mott insulator with $\nu=1+x$ the ions with lower local energies are double occupied, implying that the lower alloy subband is blocked and does not play any role. We note that for finite $U$ virtual processes leading to double occupation either in the lower $(\nu=x)$ or in the upper alloy subband $(\nu=1+x)$ are still possible, leading to the antiferromagnetic superexchange interaction. However, since the positions of the corresponding atoms are random, particle-hole symmetry is absent such that long-range antiferromagnetic order is expected to be suppressed. Of course, antiferromagnetism cannot be ruled out completely on these reasonings and its appearance in the model requires further studies.

For $U>U_{c}(\Delta)$ in the Mott-insulating state with binaryalloy disorder one may use the lowest excitation energies to distinguish two different types of insulators; namely, for $U$ $<\Delta$ an excitation must overcome the energy gap between the lower and the upper Hubbard subbands, as indicated in Fig. 2. We call this insulating state an alloy Mott insulator. 


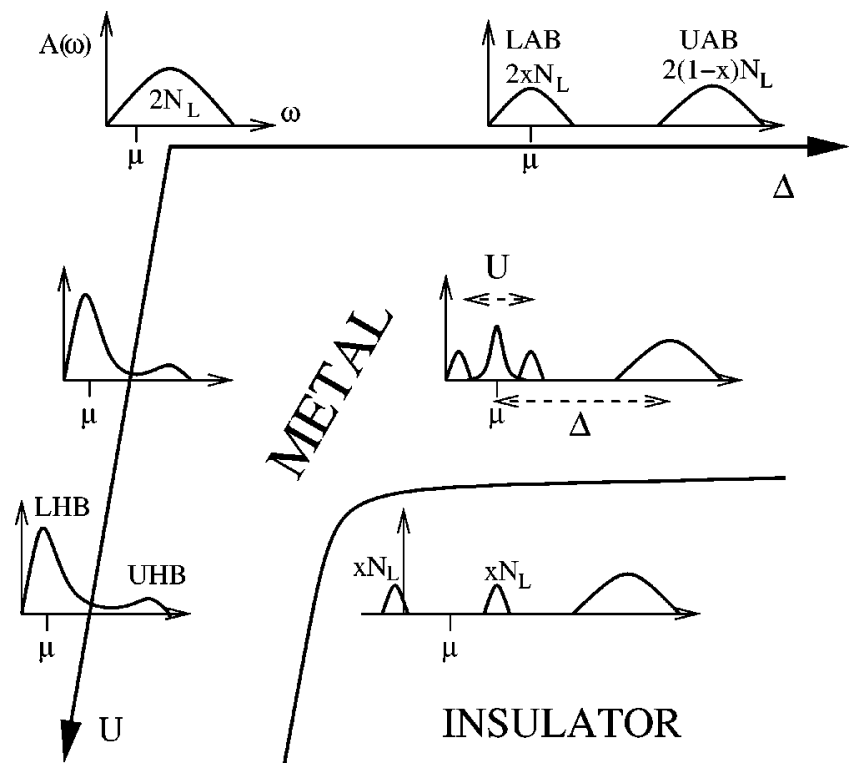

FIG. 1. Schematic plot representing the Mott-Hubbard metalinsulator transition in a correlated electron system with the binaryalloy disorder. The shapes of spectral functions $A(\omega)$ are shown for different interactions $U$ and disorder strengths $\Delta$. Increasing $\Delta$ at $U=0$ leads to splitting of the spectral function into the lower (LAB) and the upper (UAB) alloy subbands, which contain $2 x N_{L}$ and $2(1-x) N_{L}$ states, respectively. Increasing $U$ at $\Delta=0$ leads to the occurrence of lower (LHB) and upper (UHB) Hubbard subbands. The Fermi energy for filling $\nu=x$ is indicated by $\mu$. At $\nu$ $=x$ (or $\nu=1+x$, not shown in the plot) the LAB (UAB) is half filled. In this case an increase of $U$ and $\Delta$ leads to the opening of a correlation gap at the Fermi level and the system becomes a Mott insulator.

On the other hand, for $\Delta<U$ an excitation must overcome the energy gap between the lower Hubbard subband and the upper alloy-subband, as shown in Fig. 2. We call this insulating state an alloy charge transfer insulator.

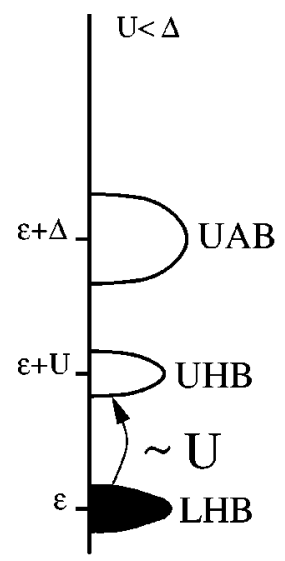

alloy Mott

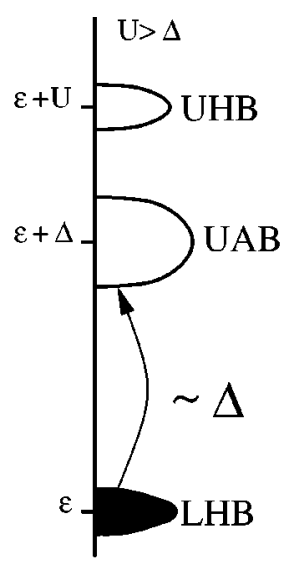

alloy charge transfer insulator
FIG. 2. Two possible insulating states in the correlated electron system with binary-alloy disorder. When $U<\Delta$ the insulating state is an alloy Mott insulator with an excitation gap in the spectrum of the order of $U$. When $U>\Delta$ the insulating state is an alloy chargetransfer insulator with an excitation gap of the order of $\Delta$.

\section{DYNAMICAL MEAN-FIELD THEORY FOR THE DISORDERED HUBBARD MODEL}

The Mott-Hubbard MIT is driven by the interaction between the particles. Since this transition occurs when the interaction energy is comparable with the single-particle energy of the electrons, there is no natural small parameter (e.g., $t$ or $U$ ) in the theory. The problem is generically nonperturbative. Moreover, when the transition appears between a paramagnetic metal and a paramagnetic insulator, there is no obvious order parameter characterizing the insulating phase. In the following the insulating state at $T=0$ is defined by the vanishing of the one-particle spectral function at the Fermi level.

The model defined by the Hamiltonian (1) is not exactly solvable for any finite number of space dimensions. However, with a proper rescaling of the hopping integral it becomes numerically solvable in infinite dimensions, i.e., within DMFT. ${ }^{6,7,29}$ For finite-dimensional systems DMFT is a self-consistent approximation scheme which takes into account local quantum fluctuations but neglects spatial correlations. Since DMFT is a nonperturbative method, it is ideally suited to study the Mott-Hubbard MIT.

To derive the DMFT equations for the problem at hand we select a single lattice site $i$ and integrate out all the electronic degrees of freedom corresponding to other sites. ${ }^{7}$ This leads to an effective single-impurity Anderson model (SIAM) Hamiltonian

$$
\begin{aligned}
H^{\mathrm{SIAM}}= & \sum_{\sigma}\left(\epsilon_{i}-\mu\right) a_{i \sigma}^{\dagger} a_{i \sigma}+U n_{i \uparrow} n_{i \downarrow}+\sum_{\mathbf{k} \sigma}\left(V_{\mathbf{k}} a_{i \sigma}^{\dagger} c_{\mathbf{k} \sigma}\right. \\
& \left.+V_{\mathbf{k}}^{*} c_{\mathbf{k} \sigma}^{\dagger} a_{i \sigma}\right)+\sum_{\mathbf{k} \sigma} \epsilon_{\mathbf{k}} c_{\mathbf{k} \sigma}^{\dagger} c_{\mathbf{k} \sigma},
\end{aligned}
$$

where $\mu$ is the chemical potential, and $V_{\mathbf{k}}$ and $\epsilon_{\mathbf{k}}$ are the hybridization matrix element and the dispersion relation for the auxiliary bath fermions $c_{\mathbf{k} \sigma}$, respectively. In the present paper the Hamiltonian (4) is solved at zero temperature using the numerical renormalization-group method. ${ }^{9-12}$ For each ionic energy $\epsilon_{i}$ we obtain the local Green function $G\left(\omega, \epsilon_{i}\right)$. The physical Green function (1) is obtained by algebraic averaging of $G\left(\omega, \epsilon_{i}\right)$ over different realizations of the disorder, ${ }^{8}$ i.e.,

$$
G(\omega)=\int d \epsilon_{i} \mathcal{P}\left(\epsilon_{i}\right) G\left(\omega, \epsilon_{i}\right)
$$

From the k-integrated Dyson equation

$$
G^{-1}(\omega)=\omega-\eta(\omega)-\Sigma(\omega)
$$

we determine the local self-energy $\Sigma(\omega)$. The function $\eta(\omega)$, called hybridization function, is defined as

$$
\eta(\omega)=\sum_{\mathbf{k}} \frac{\left|V_{\mathbf{k}}\right|^{2}}{\omega-\epsilon_{\mathbf{k}}} .
$$

The DMFT equations are closed by a Hilbert transform, relating the local Green function for a given crystallographic lattice to the self-energy, i.e., 


$$
G(\omega)=\int d \epsilon \frac{N_{0}(\epsilon)}{\omega-\epsilon-\Sigma(\omega)},
$$

where $N_{0}(\epsilon)$ is the noninteracting DOS. Equations (4)-(7) constitute a closed set of DMFT equations for the disordered Hubbard model.

We solve Eqs. (4)-(7) for a Bethe lattice with infinite connectivity. In this case the DOS is given by

$$
N_{0}(\epsilon)=\frac{2}{\pi D} \sqrt{D^{2}-\epsilon^{2}},
$$

with the bandwidth $W=2 D$. In the following we set $D$ $=1$. With the DOS (8) the Hilbert transform (7) can be calculated analytically leading to a simple algebraic relation between the local Green function $G(\omega)$ and the hybridization function $\eta(\omega)$, i.e.,

$$
\eta(\omega)=\frac{D^{2}}{4} G(\omega) .
$$

In the numerical calculations we adjust the value of the chemical potential $\mu$ so that the number of particles in the system is fixed. Hence the independent variables are $\nu, U, x$, and $\Delta$.

Since DMFT neglects short-range spatial correlations, and hence does not include effects due to backscattering of electrons on the randomly distributed ions, it cannot describe effects of Anderson localization. On the other hand, binaryalloy disorder is a particularly strong type of disorder since it even leads to band splitting-and thereby to insulating behavior-in any spatial dimension. This dominant feature, and all other effects caused by the simultaneous presence of interactions and disorder, is well captured by DMFT. In particular, the DMFT equations reduce to the equations of the coherent-potential approximation (CPA) for interaction $U$ $=0 .{ }^{30}$ The CPA method is known to be very successful in explaining single-particle properties of disordered systems, both in the case of models and realistic systems. ${ }^{19,31}$ In particular, it reproduces the alloy band splitting in binary-alloy systems. Therefore we use the DMFT to describe the MottHubbard MIT in the presence of binary-alloy disorder, and consider additional effects due to Anderson localization as secondary.

\section{NUMERICAL RESULTS FOR THE DISORDERED HUBBARD MODEL}

In the following we present our numerical results for filling factors $\nu \neq x$ and $\nu=x$ with equal concentration of $A$ and $B$ atoms, i.e., $x=1 / 2$. In particular, the ground-state phase diagram at $\nu=x$ is presented and the MIT is discussed in details.

\section{A. Interacting electrons in the alloy band splitting limit for $\nu \neq x$}

The influence of the disorder-induced alloy band splitting on the spectral function $A(\omega)$ is shown in Fig. 3 for $U=4$ and filling factor $\nu=0.3$. For vanishing disorder $(\Delta=0)$ the

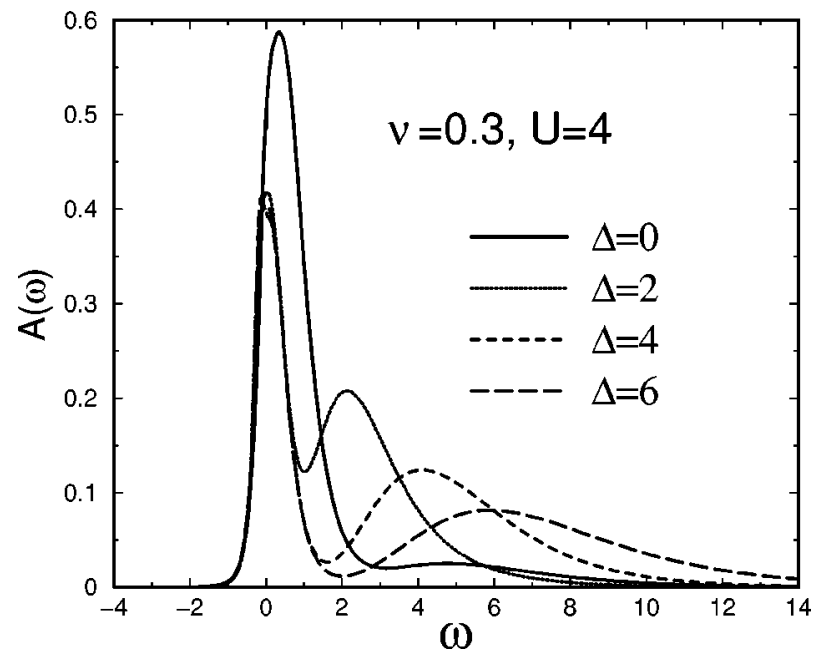

FIG. 3. Spectral function of the Hubbard model at $U=4$ and $\nu=0.3$ for different binary-alloy disorder strengths $\Delta$. The upper alloy subband splits off for $\Delta \geqslant 2$. The shape of the lower alloy subband is not significantly changed for large $\Delta$.

spectral function is composed of the lower Hubbard subband (which at this low density is merged with the quasiparticle peak) and the upper Hubbard subband around $\omega=4$. Upon increasing $\Delta$ the upper alloy subband splits off and moves to larger $\omega$. At the same time the lower alloy subband appears with a smaller number of states. Its shape and the position with respect to the Fermi energy do not change for $\Delta>2$. These results and the shape invariance of the lower alloy subband in the presence of interactions between the electrons suggest that in the $\Delta \rightarrow \infty$ limit the binary-alloy disordered Hubbard model can be effectively described by the Hubbard model in a reduced Hilbert space which contains only the states from the lower alloy subband.

\section{B. Phase diagram and Mott-Hubbard transition at filling $\boldsymbol{\nu}=\boldsymbol{x}$}

By solving numerically the DMFT equations we extracted the zero-temperature phase diagram at $\nu=x=0.5$ which is presented in Fig. 4. The curve with filled dots represents the critical interaction $U_{c 2}=U_{c 2}(\Delta)$ separating the paramagnetic metal and the paramagnetic insulator. This boundary line was determined by solving iteratively the DMFT equations using a metallic hybridization function as an initial input. It means that in solving the system of Eqs. (4)-(7) iteratively we began with the hybridization function $\eta^{(0)}(\omega)$ $\approx G^{(0)}(\omega)$ that has a nonvanishing imaginary part at $\omega=0$. The other curve (open dots) represents the boundary $U_{c 1}$ $=U_{c 1}(\Delta)$ between the metallic and insulating phases, as determined by solving the DMFT equations with an insulating hybridization function as an initial input. In this case the initial hybridization function had vanishing imaginary part at $\omega=0$. The boundary points (lines) correspond to the values of the $(U, \Delta)$ parameters where the converged spectral functions obtained from Eqs. (4)-(7) starts to have zero weight at $\omega=0$. In the inset to Fig. 4, the behavior of the spectral function at the Fermi level is shown when the metal-insulator boundary is approached from the metallic (solid lines) and 


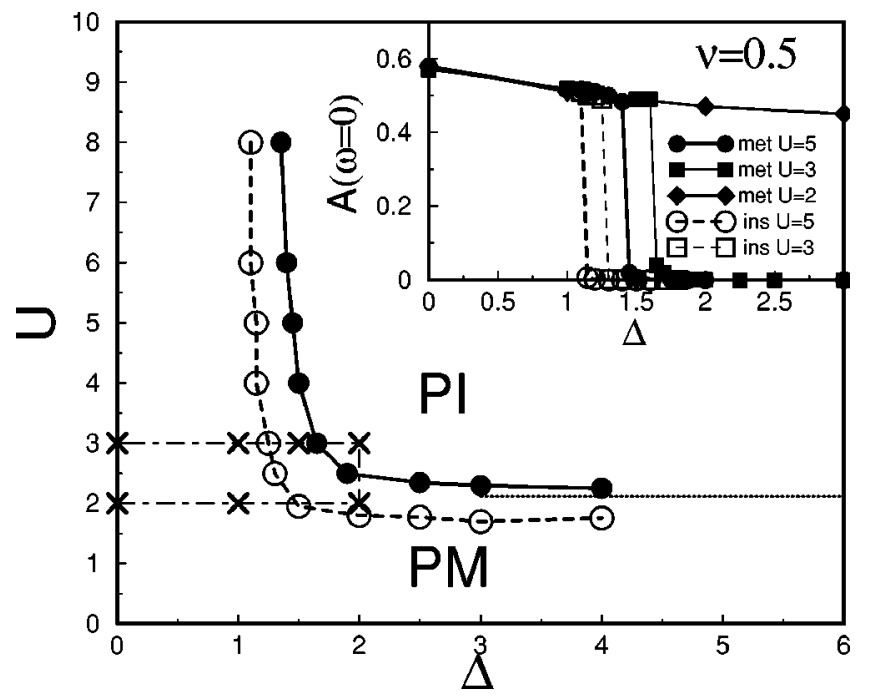

FIG. 4. Ground-state phase diagram of the Hubbard model with binary-alloy disorder at filling $\nu=x=0.5$. The filled (open) dots represent the boundary between paramagnetic metallic (PM) and paramagnetic insulating (PI) phases as determined by DMFT with the initial input given by the metallic (insulating) hybridization function. The horizontal dotted line represents $U_{c}$ obtained analytically from an asymptotic theory in the limit $\Delta \rightarrow \infty$ (see Sec. V). Stars show the points at which the explicit spectral functions are presented in Figs. 5-7. Inset: hysteresis in the spectral functions at the Fermi level obtained from DMFT with an initial metallic (insulating) host represented by filled (open) symbols and solid (dashed) lines.

from the insulating side (dashed lines), respectively. We observe hysteresis, however, at zero temperature the transition is continuous and occurs at the $U_{c}=U_{c 2}(\Delta)$ line. ${ }^{7,14} \mathrm{We}$ calculated numerically the average density of double occupied sites $d=\left\langle n_{\uparrow} n_{\downarrow}\right\rangle$ in the coexistence regime $\left(U_{c 1} \leqslant U \leqslant U_{c 2}\right)$ and found that $d$ is larger for a metallic solution. It implies that the metallic ground state is energetically more stable in this regime. ${ }^{7}$ Of course, very close to $U_{c 2}(\Delta)$ we cannot make an absolutely precise statement because of the finite numerical accuracy.

From the inset to Fig. 4 we also conclude that in the metallic phase $A(0)$, the spectral function at the Fermi level decreases with disorder but remains independent of $U$. This behavior corresponds to the "pinning" of the spectral function (Friedel sum rule) in the pure case, where $A(0)$ does not depend on the interaction $U$ between the electrons. ${ }^{32}$ Similar behavior is encountered in the disordered Hubbard model studied here. However, now $A(0)$ is reduced by the $\Delta$ dependent imaginary part of the self-energy.

In the upper panels of Figs. 5-7 we present the spectral functions for selected parameters $U$ and $\Delta$ along a path in the $(U, \Delta)$ phase diagram (Fig. 4) indicated by crosses: $U=3$ and $\Delta=0 \rightarrow 1 \rightarrow 1.5 \rightarrow 2$ (Fig. 5), $\Delta=2$ and $U=3 \rightarrow 2$ (Fig. 6), and finally, $U=2$ and $\Delta=2 \rightarrow 1 \rightarrow 0$ (Fig. 7). These spectral functions illustrate the evolution of the system within, or between, a metallic and an insulating phase when disorder and interaction are changed. In particular, in Fig. 5 we see for $U=3$ how increasing $\Delta$ from the value 0 (where the spectral function is qualitatively similar to that of the $\nu$

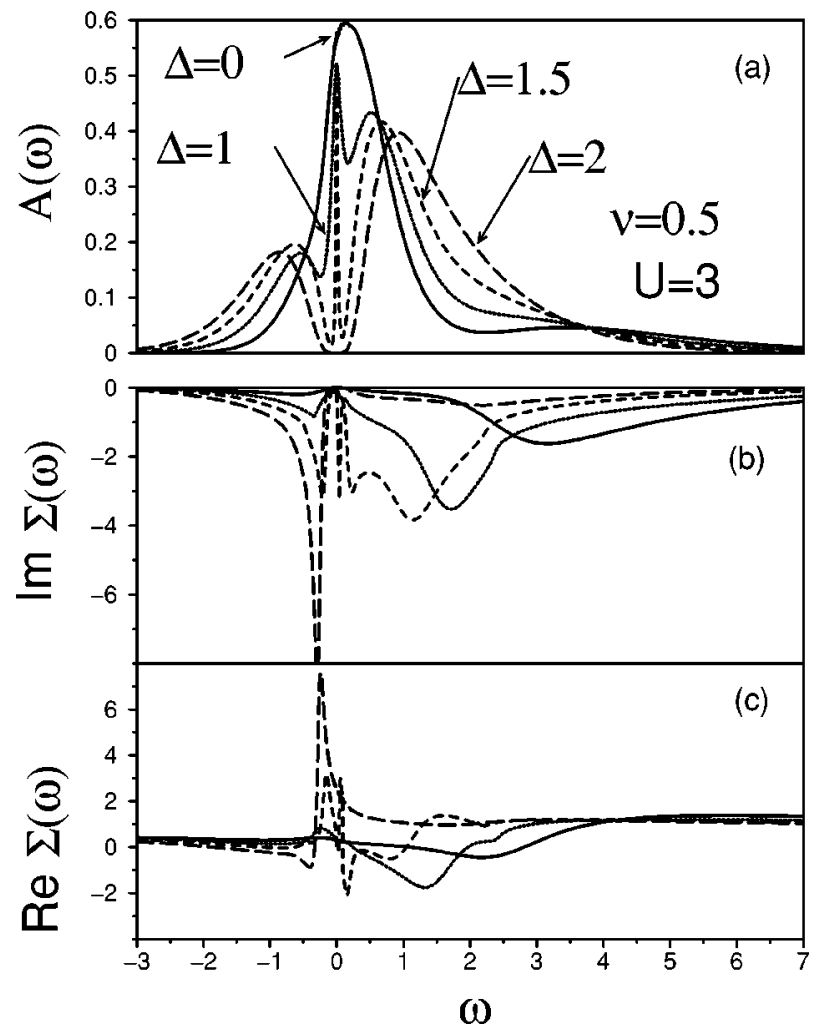

FIG. 5. (a) Spectral function, (b) imaginary part of the selfenergy, and (c) real part of the self-energy for the Hubbard model at $\nu=0.5, U=3$ and different disorder $\Delta$. As $\Delta$ increases, the quasiparticle peak appears and then vanishes, signaling a transition from a metallic to an insulating phase. In the insulating phase $(\Delta$ 2) a Mott gap is opened at $\omega=0$ with $\operatorname{Im} \Sigma(0)=0$ whereas $\operatorname{Re} \Sigma(0)$ remains finite.

$=0.3$ case, cf Fig. 3) to 1 and 1.5 leads to the splitting of the alloy subbands and the emergence of the quasiparticle peak at $\omega=0$, a feature of strong correlations between the electrons. At $\Delta=2$ the quasiparticle peak is absent and the spectral function possesses a Mott gap at the Fermi level, a feature of an insulator. Keeping $\Delta=2$ in Fig. 6 and lowering $U$ from 3 to 2 leads to a shrinking of the Mott gap and reappearance of a quasiparticle peak, characterizing a correlated metallic phase. Finally, upon lowering $\Delta$ from 2 to 0 at constant $U=2$ the alloy subbands approach each other and the quasiparticle peak merges with the lower Hubbard subband as presented in Fig. 7.

In addition to the spectral functions, the imaginary and real parts of the self-energy, calculated by the method presented in the Appendix, are shown in panels (b) and (c) in Figs. 5-7. In the metallic phase with $\Delta>0$, two important features of the self-energy should be noted: (i) the imaginary part of the self-energy at the Fermi level is finite, i.e., $\operatorname{Im} \Sigma(\omega=0)<0$ and (ii) the real part of the self-energy at the Fermi level has a negative slope, i.e., $\partial \operatorname{Re} \Sigma(\omega=0) / \partial \omega<0$. While the former feature (caused by disorder even in the presence of the local interaction) can be observed within the perturbation expansion with respect to small $\Delta$, the latter is surprising since at $U=0$ the slope is always positive for any $\Delta>0 .{ }^{33}$ 

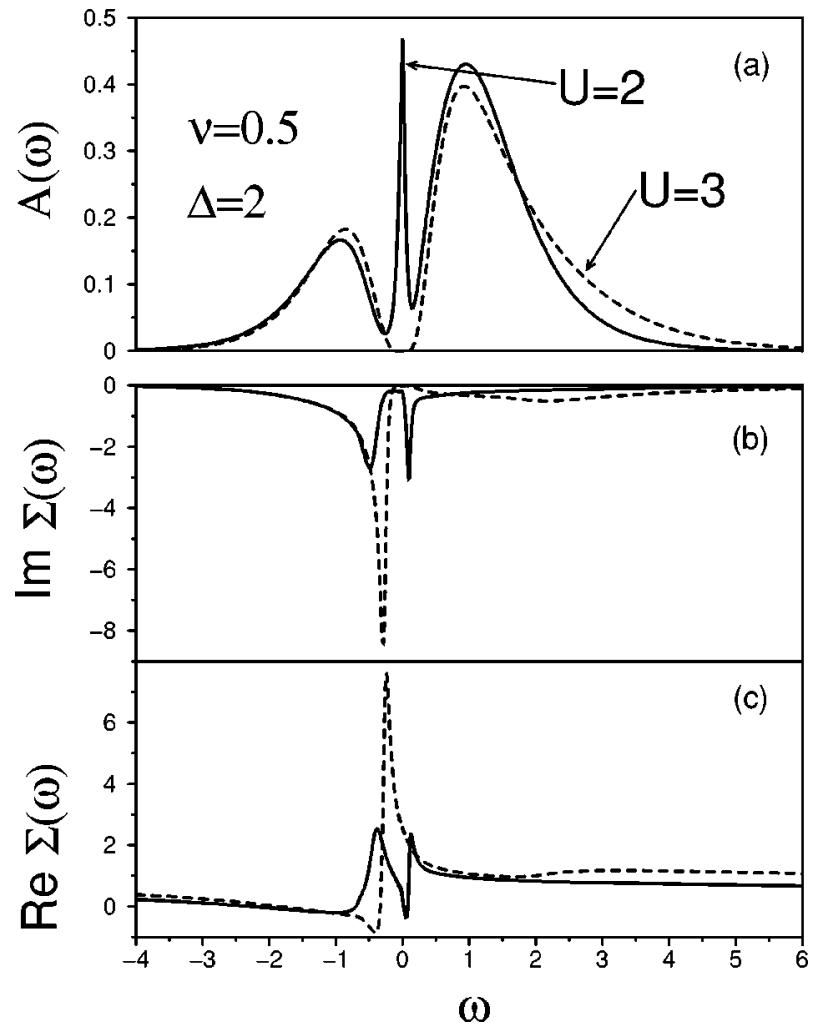

FIG. 6. (a) Spectral function, (b) imaginary part of the selfenergy, and (c) real part of the self-energy for the Hubbard model at $\Delta=2$ and $U=3$ and 2 .

At the MIT the behavior of the self-energy changes. In particular, the imaginary part becomes vanishingly small at $\omega=0$ whereas the real part is finite. These results imply that the mechanism for opening a correlation (Mott) gap at this MIT transition is different from that in the pure Hubbard model with particle-hole symmetry, ${ }^{7,14}$ namely, consider the spectral function at the Fermi level, which is expressed in terms of the self-energy:

$$
A(0)=-\frac{1}{\pi} \int d \epsilon N_{0}(\epsilon) \frac{\operatorname{Im} \Sigma(0)}{[\epsilon-\operatorname{Re} \Sigma(0)]^{2}+[\operatorname{Im} \Sigma(0)]^{2}}
$$

Since we obtained numerically that at the Fermi level the imaginary part of the self-energy vanishes and the real part is larger than the bandwidth $W$, we find $A(0)=N_{0}[\operatorname{Re} \Sigma(0)]$ $=0$. This result is in contrast to the Mott-Hubbard MIT in the pure Hubbard model at half filling with particle-hole symmetry. In this last case the opening of a correlation gap at $T=0$ is due to the formation of a $\delta$-like singularity in the imaginary part of the self-energy at the Fermi level. The real part of $\Sigma(\omega)$ has a $1 / \omega$ divergence at this point, consistent with the Kramers-Kronig relations. In the disordered case we do not see such a behavior of $\operatorname{Re} \Sigma(\omega)$, which implies that lifting of the particle-hole symmetry due to finite disorder and noninteger filling affects the way how the gap is opened in the Mott insulator.

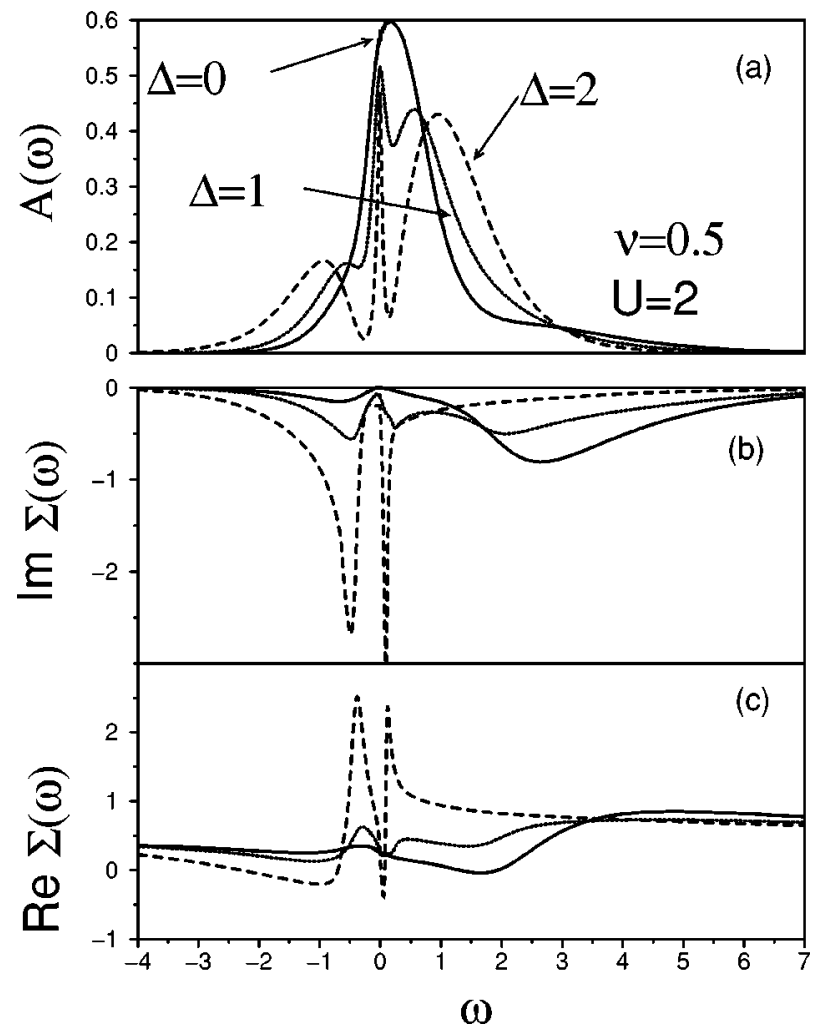

FIG. 7. (a) Spectral function, (b) imaginary part of the selfenergy, and (c) real part of the self-energy for the Hubbard model at $U=2$ and different disorder $\Delta$. Upon increasing $\Delta$ the alloy subbands are split and a quasiparticle resonance emerges between the Hubbard subbands.

\section{ASYMPTOTIC LIMIT $\Delta \rightarrow \infty$}

Our understanding of the Hubbard model with binaryalloy disorder is based on the fact that the lower and upper alloy subbands are split at large $\Delta$. We now show that for $\Delta \rightarrow \infty$ the upper (or lower) alloy subband can be neglected and the problem can be mapped onto a low-energy subspace of the full Hilbert space. Effectively, the correlated and binary-alloy disordered electronic system is represented by a correlated pure system with renormalized parameters.

\section{A. Mapping of the Hilbert space}

We consider the case $\Delta \gg \max (U, W)$. Then the Hilbert space can be divided into two subspaces $A$ and $B$ consisting of ions with energies $\epsilon_{A}$ and $\epsilon_{B}$, respectively. We denote the projection operator onto the $A$ subspace by $\hat{P}$ and the projection operator onto the $B$ subspace by $\hat{Q}=1-\hat{P} \cdot{ }^{35,36}$ The Schrödinger equation can be decomposed as

$$
\begin{aligned}
& (E-\hat{P} H \hat{P}) \hat{P}|\Psi\rangle=\hat{P} H \hat{Q} \hat{Q}|\Psi\rangle, \\
& (E-\hat{Q} H \hat{Q}) \hat{Q}|\Psi\rangle=\hat{Q} H \hat{P} \hat{P}|\Psi\rangle,
\end{aligned}
$$

where $|\Psi\rangle$ is a many-body eigenstate of the Hamiltonian (1) with eigenvalue $E$. Solving this set of equations, the effective Hamiltonian of the lower alloy subband is formally given by 


$$
H_{\mathrm{eff}}=\hat{P} H \hat{P}-\hat{P} H \hat{Q} \frac{1}{\hat{Q} H \hat{Q}} \hat{Q} H \hat{P} .
$$

The mapping implies that the number of lattice sites corresponding to the $A$ subspace is equal to the number of $\epsilon_{A}$ ions, i.e., $N_{A}=x N_{L}$. Therefore, if we restrict ourselves to this low-energy many-body subspace $\hat{P}|\Psi\rangle$, and consider only the effective Hamiltonian $H_{\text {eff }}$, the filling factor can be replaced by $\nu^{*}=\nu / x$.

The interaction $U$ is not changed by the projection of the Hilbert space because it is a local quantity. However, the bandwidth of the lower alloy subband is renormalized because the number of nearest neighbors with on-site energy $\epsilon_{A}$ is reduced. In order to estimate how the bandwidth renormalizes we consider the Bethe lattice with a finite coordination number $z$ and calculate the second moment $\mu_{i}^{(2)}$ $=\left\langle A\left|H_{0}^{2}\right| A\right\rangle$ for the noninteracting Hamiltonian $H_{0}$ $=t \sum_{i, \delta} a_{i}^{\dagger} a_{i+\delta}$ with nearest-neighbor hopping. For a given lattice site $A$ we can have $z_{A}$ neighbors with energies $\epsilon_{A}$, where $0 \leqslant z_{A} \leqslant z$. It is easy to show that the second moment at site $A$ is $\mu_{i}^{(2)}\left(z_{A}\right)=z_{A} t^{2}$. The probability distribution of $z_{A}$ is given by

$$
\mathcal{P}\left(z_{A}\right)=\left(\begin{array}{c}
z \\
z_{A}
\end{array}\right) x^{z_{A}}(1-x)^{z-z_{A}} .
$$

Therefore we find the average moment $\left\langle\mu^{(2)}\right\rangle:^{24}$

$$
\left\langle\mu^{(2)}\right\rangle=\frac{1}{N_{A}} \sum_{i=1}^{N_{A}} \mu_{i_{A}}^{2}\left(z_{A}\right) \mathcal{P}\left(z_{A}\right)=z t^{2} x .
$$

For a Bethe lattice with coordination number $z$ tending to infinity we rescale $t \rightarrow t^{*}=t / \sqrt{z}$. We then find that the second moment is $\left\langle\mu^{(2)}\right\rangle=t^{* 2} x$. It means that the bandwidth, as measured by the second moment, is reduced in the effective Hamiltonian $H_{\text {eff }}$ by the factor $\sqrt{x}$.

\section{B. Critical interaction $U_{c}$}

The approximate value of the critical interaction for the occurrence of the Mott transition can be found analytically within the linearized DMFT, where the full DMFT problem is mapped onto the two-site SIAM with self-consistency conditions. ${ }^{34}$ For the pure Hubbard model it was shown that the critical interaction has the value $U_{c}=6 \sqrt{\mu^{(2)}}$. From the results in the preceding section we find that the critical interaction for the MIT in the strong disorder limit $\Delta \rightarrow \infty$ is given by $U_{c}=6 t^{*} \sqrt{x}$. For parameters used in our calculations $t^{*}$ $=0.5$ and $x=0.5$, we obtain $U_{c}=3 / \sqrt{2}$. This value is shown by the dotted line in Fig. 4. The agreement between our numerical calculation and this estimate of $U_{c}$ is surprisingly good.

\section{CONCLUSIONS}

In this paper we studied the Mott-Hubbard transition in a correlated electronic system with binary-alloy disorder. By numerically solving the DMFT equations at $T=0$ we showed that for filling factor $\nu=x$ a MIT occurs when the disorder $\Delta$ or the interaction $U$ is increased. Regarding the excitation spectrum for the electrons we introduced the notion of the "alloy Mott insulator" for $U<\Delta$ and of the "alloy charge-transfer insulator" for $U>\Delta$. This classification is analogous to the Zaanen-Sawatzky-Allen scheme for twoband Hubbard systems. ${ }^{37}$ In our case however, the role of the oxygen band is played by the upper alloy subband. We also found hysteresis upon approaching the metal-insulator boundary, depending on the initial conditions imposed in the iterative solution of the DMFT equations. It shows that hysteresis is a generic feature of the MIT in pure and in binaryalloy disordered systems within the DMFT scenario. We also found that the opening of a Mott gap is associated with the disappearance of the imaginary part of the self-energy at the Fermi level. Finally we discussed the analytical theory, valid in the alloy band splitting limit, and showed that the Hubbard model with binary-alloy disorder can be mapped onto an effective Hubbard Hamiltonian with renormalized bandwidth and filling factor. The estimated critical interaction $U_{c}$ in this asymptotic theory agrees very well with the numerical results. We note that the Hubbard model with a binary-alloy distributed $U$ can also have an insulating ground state at noninteger fillings. ${ }^{38}$

Our study of the Mott-Hubbard MIT was limited to the Hubbard model with a nondegenerate orbital. A similar phase transition should be expected in the Hubbard model with orbital degeneracy. An important question is, however, whether one can find a physical system where such a transition is realized. For binary alloys this might be a very demanding task because the predicted MIT requires fine tuning of the filling factor with concentration of the alloy elements as well as special values for the interaction and the disorder splitting. At present we do not know which alloy system would be the best candidate for realizing the predicted MottHubbard MIT.

The most promising candidates for experimental realization may come from systems with fermions moving on artificial lattices. Creating a lattice with a binary-alloy disorder seem to be possible either with a matrix of quantum dots with two different sizes, ${ }^{39}$ or with optical lattices, where counterpropagating laser beams can be used to trap fermionic atoms. ${ }^{40}$ In the latter case, with proper selection of laser light and physical boundaries one can obtain at least quasidisordered systems ${ }^{41}$ where a binary-alloy-like distribution is possible. From the point of view of our theory, where Anderson localization is not included, quasirandomness of the optical lattice is not a major limitation as it yields the effective alloy band splitting which is crucial for our calculation. Since in the present Mott insulator the long-range ordering is suppressed due to the disorder, such a system might be very useful to study the ground state of, and excited states in, paramagnetic Mott insulators.

\section{ACKNOWLEDGMENTS}

We thank R. Bulla for helpful discussions and B. Velický for valuable correspondence. This work was supported in part by the Sonderforschungsbereich 484 of the Deutsche Forschungsgemeinschaft (DFG). Financial support of K.B. 
through Grant No. KBN-2 P03B 08224 and of W.H. through the DFG is also gratefully acknowledged.

\section{APPENDIX: CALCULATION OF THE SELF-ENERGY}

The self-energy can, in principle, be calculated directly from a Dyson equation $\Sigma(\omega)=G^{-1}(\omega)-\omega+\eta(\omega)$. However, Bulla et al. ${ }^{11}$ have proposed a different approach, namely, to use a two-particle correlation function to determine the self-energy. In this way certain systematic errors, leading to inaccurate spectral weights, are canceled. In this appendix we show how to generalize this method to find the self-energy in the correlated system with binary-alloy disorder. With a new interpretation of the CPA equation one can use two-particle correlation functions to determine the single-particle self-energy. Our method can be applied to an arbitrary DOS and, as we checked independently, leads to better convergence of the DMFT equations due to apparent error cancellations.

Within the DMFT, the disordered Hubbard model is mapped onto a single-impurity Anderson model which contains the local ionic energy $\epsilon_{i}$ as a parameter. For each $\epsilon_{i}$ the Anderson model is solved independently. It yields the local $\epsilon_{i}$-dependent Green function $G\left(\omega, \epsilon_{i}\right)=\left\langle\left\langle a_{i \sigma} \mid a_{i \sigma}^{\dagger}\right\rangle\right\rangle$. In addition, for each $\epsilon_{i}$ we can find a two-particle Green function

$$
F\left(\omega, \epsilon_{i}\right)=\left\langle\left\langle a_{i \sigma} a_{i \bar{\sigma}}^{\dagger} a_{i \bar{\sigma}} \mid a_{i \sigma}^{\dagger}\right\rangle\right\rangle .
$$

On the other hand, the equation of motion for $G\left(\omega, \epsilon_{i}\right)$ obtained from the $\epsilon_{i}$ dependent SIAM is

$$
\left(\omega-\epsilon_{i}\right) G\left(\omega, \epsilon_{i}\right)-U F\left(\omega, \epsilon_{i}\right)-\eta(\omega) G\left(\omega, \epsilon_{i}\right)=1,
$$

which can be rewritten as

$$
[\omega-\eta(\omega)] G\left(\omega, \epsilon_{i}\right)=1+V_{i}(\omega) G\left(\omega, \epsilon_{i}\right),
$$

where we define a complex and frequency dependent (dynamical) scattering potential

$$
V_{i}(\omega) \equiv \epsilon_{i}+U \frac{F\left(\omega, \epsilon_{i}\right)}{G\left(\omega, \epsilon_{i}\right)} .
$$

From a formal point of view Eq. (A3) looks like a singleparticle equation for a Green function $G\left(\omega, \epsilon_{i}\right)$ with random potential $V_{i}(\omega)$. The average Green function $G(\omega)$ is found within the CPA by demanding that the average of the transfer matrix, given by

$$
T_{i}(\omega)=\frac{\epsilon_{i}+U \frac{F\left(\omega, \epsilon_{i}\right)}{G\left(\omega, \epsilon_{i}\right)}-\Sigma(\omega)}{1-\left[\epsilon_{i}+U \frac{F\left(\omega, \epsilon_{i}\right)}{G\left(\omega, \epsilon_{i}\right)}-\Sigma(\omega)\right] G(\omega)}
$$

with self-energy $\Sigma(\omega)$, vanishes, i.e., $\int d \epsilon_{i} \mathcal{P}\left(\epsilon_{i}\right) T_{i}(\omega)=0$.

For binary-alloy disorder the equation for the self-energy can be solved analytically, leading to

$$
\Sigma(\omega)=\frac{1}{2}\left[V_{1}(\omega)+V_{2}(\omega)-\frac{1}{G(\omega)} \pm \Xi(\omega)\right],
$$

where

$$
\begin{aligned}
\Xi(\omega)= & \left(\left[V_{1}(\omega)+V_{2}(\omega)-\frac{1}{G(\omega)}\right]^{2}-4\left[V_{1}(\omega) V_{2}(\omega)\right.\right. \\
& \left.\left.-x \frac{V_{1}(\omega)}{G(\omega)}-(1-x) \frac{V_{2}(\omega)}{G(\omega)}\right]\right)^{1 / 2} .
\end{aligned}
$$

Causality of the Green function requires that the sign in Eq. (A6) has to be properly chosen: (i) at $\omega \rightarrow-\infty(+\infty)$ the physical solution of Eq. (A6) has a $(-)[(+)]$ sign and (ii) the change of the sign happens an odd number of times at frequencies $\omega_{0}$ for which $\operatorname{Im} \Xi\left(\omega_{0}\right)=0$.
${ }^{1}$ N.F. Mott, Proc. Phys. Soc., London, Sect. A 62, 416 (1949); Metal-Insulator Transitions, 2nd ed. (Taylor and Francis, London, 1990).

${ }^{2}$ F. Gebhard, The Mott Metal-Insulator Transition (Springer, Berlin, 1997).

${ }^{3}$ T. Paiva and R.R. dos Santos, Phys. Rev. B 58, 9607 (1998); J. Silva-Valencia, E. Miranda, and R.R. dos Santos, ibid. 65, 115115 (2002).

${ }^{4}$ D.-H. Lee and S.A. Kivelson, Phys. Rev. B 67, 024506 (2003).

${ }^{5}$ K. Byczuk, M. Ulmke, and D. Vollhardt, Phys. Rev. Lett. 90, 196403 (2003).

${ }^{6}$ D. Vollhardt, in Correlated Electron Systems, edited by V.J. Emery (World Scientific, Singapore, 1993), Vol. 9, p. 57.

${ }^{7}$ A. Georges, G. Kotliar, W. Krauth, and M.J. Rozenberg, Rev. Mod. Phys. 68, 13 (1996).

${ }^{8}$ M. Ulmke, V. Janiš, and D. Vollhardt, Phys. Rev. B 51, 10411 (1995).

${ }^{9}$ K.G. Wilson, Rev. Mod. Phys. 47, 773 (1975).

${ }^{10}$ T.A. Costi, A.C. Hewson, and V. Zlatić, J. Phys.: Condens. Matter 6, 2519 (1994).
${ }^{11}$ R. Bulla, A.C. Hewson, and Th. Pruschke, J. Phys.: Condens. Matter 10, 8365 (1998).

${ }^{12}$ W. Hofstetter, Phys. Rev. Lett. 85, 1508 (2000).

${ }^{13}$ M.J. Rozenberg, G. Kotliar, and X.Y. Zhang, Phys. Rev. B 49, 10181 (1994).

${ }^{14}$ R. Bulla, Phys. Rev. Lett. 83, 136 (1999).

${ }^{15}$ E. Müller-Hartmann, in Proceedings of the V Symposium Phys. of Metals, edited by E. Talik and J. Szade (Silesian University, Poland, 1991), p. 22.

${ }^{16}$ M. Ulmke, Eur. Phys. J. B 1, 301 (1998)

${ }^{17}$ D. Vollhardt, N. Blümer, K. Held, and M. Kollar, in BandFerromagnetism, edited by K. Baberschke, M. Donath, and W. Nolting, Lecture Notes in Physics Vol. 580 (Springer, Berlin, 2001), p. 191.

${ }^{18}$ S. Kirkpatrick, B. Velicky̌, and H. Ehrenreich, Phys. Rev. B 1, 3250 (1970).

${ }^{19}$ A. Gonis, Green's Functions for Ordered and Disordered Systems (North-Holland, Amsterdam, 1992).

${ }^{20}$ P.W. Anderson, Phys. Rev. 109, 1492 (1958).

${ }^{21}$ E.N. Economou, S. Kirkpatrick, Morrel H. Cohen, and T.P. Egg- 
arter, Phys. Rev. Lett. 25, 520 (1970).

${ }^{22}$ S. Kirkpatrick and T.P. Eggarter, Phys. Rev. B 6, 3598 (1972).

${ }^{23}$ B.Y. Tong and M.M. Pant, Phys. Rev. B 21, 574 (1980).

${ }^{24}$ G.G. Naumis, C. Wang, and R.A. Barrio, Phys. Rev. B 65, 134203 (2002).

${ }^{25}$ M.J. Rozenberg, X.Y. Zhang, and G. Kotliar, Phys. Rev. Lett. 69, 1236 (1992).

${ }^{26}$ X.Y. Zhang, M.J. Rozenberg, and G. Kotliar, Phys. Rev. Lett. 70, 1666 (1993).

${ }^{27}$ G. Kotliar and H. Kajueter, Phys. Rev. B 54, 14221 (1996).

${ }^{28}$ H. Kajueter and G. Kotliar, Int. J. Mod. Phys. B 11, 729 (1997).

${ }^{29}$ W. Metzner and D. Vollhardt, Phys. Rev. Lett. 62, 324 (1989).

${ }^{30}$ R. Vlaming and D. Vollhardt, Phys. Rev. B 45, 4637 (1992).

${ }^{31}$ See, e.g., K. Koepernik, B. Velický, R. Hayn, and H. Eschrig, Phys. Rev. B 55, 5717 (1997); J. Tobola, L. Jodin, P. Pecheur, H. Scherrer, G. Venturini, B. Malaman, and S. Kaprzyk, ibid. 64, 155103 (2001).

${ }^{32}$ E. Müller-Hartmann, Z. Phys. B: Condens. Matter 76, 211 (1989); Int. J. Mod. Phys. B 3, 2169 (1989).

${ }^{33}$ M.S. Laad, L. Craco, and E. Müller-Hartmann, Phys. Rev. B 64,
195114 (2001).

${ }^{34}$ R. Bulla and M. Potthoff, Eur. Phys. J. B 13, 257 (2000).

${ }^{35}$ K.A. Chao, J. Spalek, and A.M. Oleś, Phys. Status Solidi B 84, 747 (1977).

${ }^{36}$ Explicitly, one finds $\hat{P}|\Psi\rangle=\Pi_{l} \Theta\left(\Lambda-\epsilon_{l}+\Delta / 2\right) \sum A_{l_{1} \ldots l_{N}} a_{l_{1} \sigma_{l_{1}}^{\dagger}}^{\dagger} \ldots$ $a_{l_{N} \sigma_{N}}^{\dagger}|0\rangle$, where $\Theta$ is a step function and $\Lambda \ll \Delta$ is a high-energy cutoff.

${ }^{37}$ J. Zaanen, G.A. Sawatzky, and J.W. Allen, Phys. Rev. Lett. 55, 418 (1985).

${ }^{38}$ M. Ulmke, P.J.H. Denteneer, R.T. Scalettar, and G. Zimanyi, Europhys. Lett. 42, 655 (1998).

${ }^{39}$ O.L. Lazarenkova and A.A. Balandin, Phys. Rev. B 66, 245319 (2002); M. Koskinen, S.M. Reimann, and M. Manninen, Phys. Rev. Lett. 90, 066802 (2003).

${ }^{40}$ W. Hofstetter, J.I. Cirac, P. Zoller, E. Demler, and M.D. Lukin, Phys. Rev. Lett. 89, 220407 (2002).

${ }^{41}$ P. Horak, J.Y. Courtois, and G. Grynberg, Phys. Rev. A 58, 3953 (1998); R. Roth and K. Burnett, J. Opt. B: Quantum Semiclassical Opt. 5, S50 (2003). 